\title{
Sure, We Can Image That! An Attempt to Image the Edge of a Growing Bacterial Colony
}

\author{
Garnet Martens ${ }^{1}$ and Kevin Hodgson ${ }^{1}$ \\ ${ }^{1}$ Bioimaging Facility, University of British Columbia, Vancouver, BC, CANADA
}

A while back we received a request to image a growing bacteria colony. Now given that we have years of live-cell imaging experience, we imagined the conditions required to collect data from a bacterial colony would be easier to control than that of mammalian cells. Bacteria don't move too much and they don't need $\mathrm{CO} 2$.

Our initial efforts using LSCM (Fig. 1) seemed to indicate we were on the right track but then things turned completely upside down. Almost all our efforts were in vain. Applying a coverslip to obtain high enough resolution to see individual cells (Fig. 1) created an anaerobic environment prohibiting cell growth. We tried overnight imaging with a spinning disk confocal (Fig. 2) several times and we retried imaging with a LSCM (weird drying artifacts in the agar). We had some initial success imaging with a GoPro camera mounted inside the incubator (Fig. 3). Some of the best data came from mounting a DSLR/macro lens inside a custom chamber (Fig. 4) and imaging the plate inside the incubator (Fig. 5).

What we really wanted to see were the individual cells at the edge of the growing colony. So, what do cunning microscopists do when the equipment in your facility cannot do the job? We ask for an equipment demo, that's what we do. After a few trials we were finally able to image some growing colonies using a relatively new instrument called the Incucyte. We are still working out the bugs (pun intended) of this system but the combination of phase and fluorescence is finally giving us some decent data (Fig. 6a). The first conclusion we have found is that the edge of the colony exists as a single cell layer at least 10 cells deep (white arrow in Fig 6a and b). Moving toward the center of the colony the cells begin to pile up in a second step, then a third step until finally they are so deep that the colony looks homogenous. At the end of the day we have learned some valuable lessons in patience and we will keep this project in mind the next time the thought "sure, we can image that" comes to mind. 


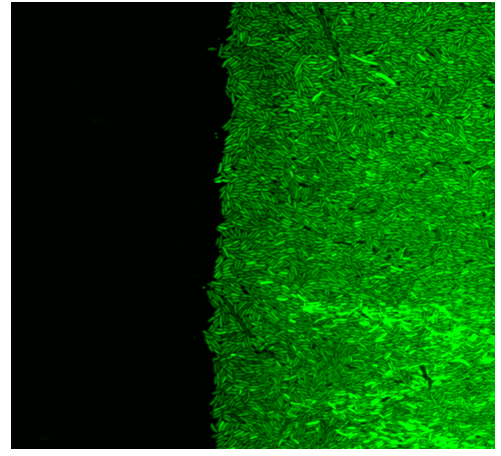

Fig. 1 LSCM edge of GFP expressing E. coli colony. Imaged with 60X oil.

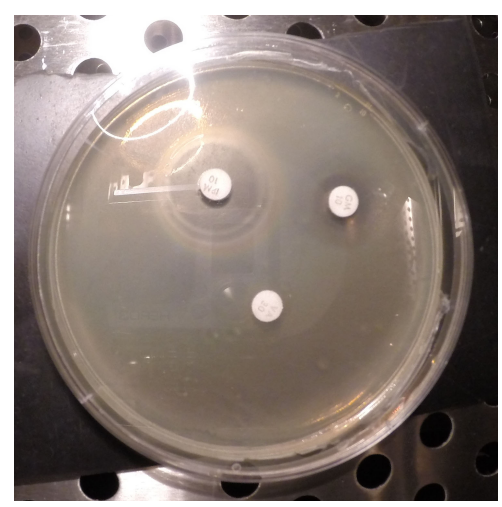

Fig. 3 Lawn of E. coli after exposure to three different antibiotics. Imaged with a GoPro Hero 3+.

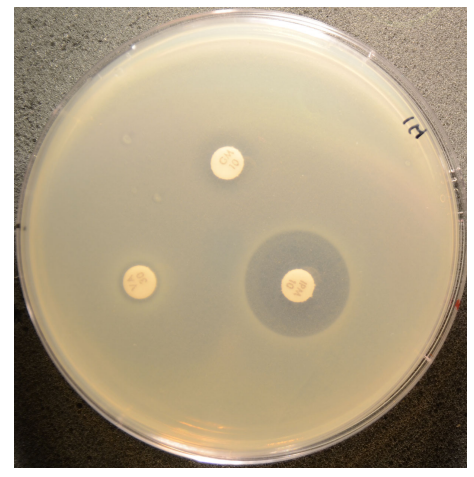

Fig. 5 Lawn of E. coli after 3- hour exposure to antibiotics. Imaged with a Nikon D7000 at $35 \mathrm{~mm}$

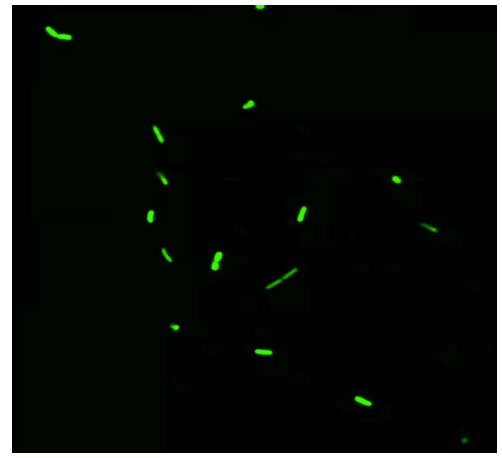

Fig.2 Individual E. coli that simply refused to grow into colony forming units. Imaged with spinning disk confocal

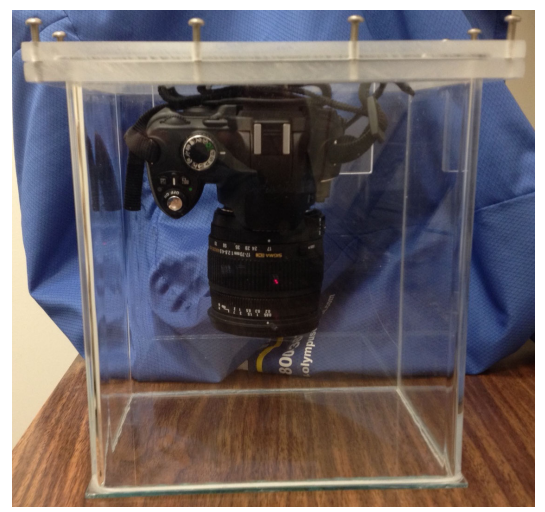

Fig. 4 Our custom chamber to allow macro photography within our tissue incubator.
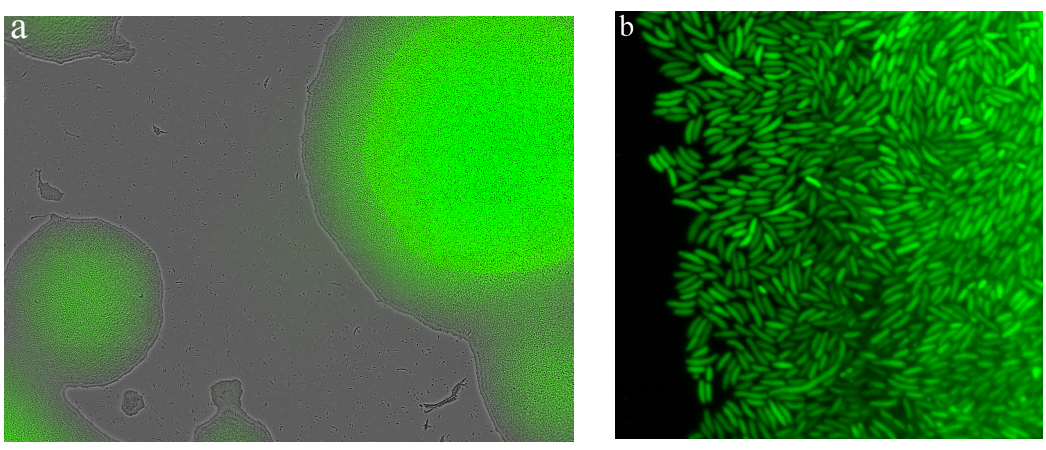

Fig. 6 Growing GFP expressing E. coli colonies. Imaged with the Incucyte (20X) (a) and LSCM (60X oil lens with $2 \mathrm{X}$ digital zoom) (b) 